\title{
Papers
}

\section{Depression and anxiety in women with early breast cancer: five year observational cohort study}

\author{
Caroline Burgess, Victoria Cornelius, Sharon Love, Jill Graham, Michael Richards, Amanda Ramirez
}

\begin{abstract}
Objective To examine the prevalence of, and risk factors for, depression and anxiety in women with early breast cancer in the five years after diagnosis.

Design Observational cohort study.

Setting NHS breast clinic, London.

Participants 222 women with early breast cancer: 170 (77\%) provided complete interview data up to either five years after diagnosis or recurrence.

Main outcome measures Prevalence of clinically important depression and anxiety (structured psychiatric interview with standardised diagnostic criteria) and clinical and patient risk factors, including stressful life experiences (Bedford College life events and difficulties schedule).

Results Nearly $50 \%$ of the women with early breast cancer had depression, anxiety, or both in the year after diagnosis, $25 \%$ in the second, third, and fourth years, and $15 \%$ in the fifth year. Point prevalence was 33\% at diagnosis, falling to $15 \%$ after one year. $45 \%$ of those with recurrence experienced depression, anxiety, or both within three months of the diagnosis. Previous psychological treatment predicted depression, anxiety, or both in the period around diagnosis (one month before diagnosis to four months after diagnosis). Longer term depression and anxiety, were associated with previous psychological treatment, lack of an intimate confiding relationship, younger age, and severely stressful non-cancer life experiences. Clinical factors were not associated with depression and anxiety, at any time. Lack of intimate confiding support also predicted more protracted episodes of depression and anxiety.

Conclusion Increased levels of depression, anxiety, or both in the first year after a diagnosis of early breast cancer highlight the need for dedicated service provision during this time. Psychological interventions for women with breast cancer who remain disease free should take account of the broader social context in which the cancer occurs, with a focus on improving social support.
\end{abstract}

\section{Introduction}

Earlier diagnosis of breast cancer in women and the use of systemic adjuvant therapy have increased the likelihood of long term, disease free survival. ${ }^{1}$ Although the psychological effect of diagnosis and treatment is well documented, less is known about the prevalence of, and risk factors for, clinically important depression and anxiety after the first year. Overall, women who remain free of breast cancer seem to have levels of functioning and quality of life that are comparable to those of the general female population, although those who receive systemic adjuvant chemotherapy may do less well. ${ }^{2}$ These findings from a questionnaire based study are encouraging, but they do not tell us about levels of clinically important depression and anxiety that might benefit from a psychological intervention. A better understanding of clinically important depression and anxiety among women who are in long term remission from breast cancer would inform appropriate service provision.

We assessed the prevalence of clinically important depression and anxiety in women with breast cancer in the five years after diagnosis using a clinical interview, and we identified risk factors for depression and anxiety over that time. We also examined the prevalence of, and risk factors for, depression and anxiety around recurrence.

\section{Participants and methods}

We invited to participate in our study a consecutive series of 222 women, aged 60 years or younger, with a diagnosis of early breast cancer at Guy's Hospital breast unit between May 1991 and July 1994. Exclusion criteria included previous or concomitant malignancy, pregnancy, and age over 60. The women were treated surgically by lumpectomy, axillary clearance, and radiotherapy or by modified radical mastectomy followed by adjuvant therapy as determined by the women's biological risk factors.

We recruited women about eight weeks after diagnosis. They were interviewed five months after diagnosis and then every 18 months up to five years. Women who had a recurrence took part in a final interview about eight weeks after diagnosis. The interviews were audiotaped.

\section{Instruments}

We elicited psychiatric symptoms using a shortened version of the structured clinical interview (SCID), ${ }^{3}$ focusing on symptoms of depression and anxiety. At the five month interview we collected data on depression and anxiety for the period one month before diagnosis to five months after diagnosis. Data on depression and anxiety were also collected at 21, 39, and 60 months for the period between interviews. Using standardised diagnostic criteria from the Diagnostic and Statistical Manual of Mental Disorders, third edition, revised, we classified the women as full case, borderline case, or non-case for anxiety, depression, or both (see bmj.com). ${ }^{4}$ We calculated the point prevalence as the percentage of women with depression, anxiety, or both in the month before a given time point. The annual period prevalence

Diagnostic criteria for depression and anxiety are on bmj.com 
was the percentage of women who had at least one episode of depression, anxiety, or both over a one year period.

Severely stressful non-cancer related life experiences were assessed using the Bedford College life events and difficulties schedule (discrete life events and ongoing difficulties). ${ }^{5}$ At the five month interview we collected data for severely stressful life experiences for the period one month before diagnosis to five months after diagnosis. Data on severely stressful life experiences were also collected at 21,39, and 60 months for the intervening period.

We classified an intimate confiding relationship as one with a cohabiting partner (with or without sexual intimacy). ${ }^{6}$ Previous psychological treatment was defined as any psychological treatment from a general practitioner or as a hospital inpatient or outpatient.

\section{Statistical analysis}

We assessed the effects of the potential risk factors for three periods: around diagnosis (one month before diagnosis to four months after diagnosis), medium term (four months to two years after diagnosis), and longer term (two years to five years after diagnosis). These periods were chosen because most of the initial episodes of depression and anxiety were resolved at around four months, with a further fall-off after two years.

We treated age as a continuous variable. Other patient factors were coded as absent or present. The effect of a severely stressful life experience on a patient was taken to last six months. If more than one severe life event or more than one difficulty was experienced simultaneously, then the life event or difficulty was taken to be present from the earliest start date to the latest finish date.

Patients were classified into two groups for the phase around diagnosis: those who had depression, anxiety, or both during this period and those who did not. We used a logistic regression model to investigate potential risk factors for depression and anxiety.

As depression and anxiety varied over time for the medium and longer term periods, we investigated potential risk factors using Cox's proportional hazards model, with depression, anxiety, or both as the event. Patients were censored at death or recurrence. Severely stressful life experiences were treated as time varying covariates, with all other patient related variables determined at diagnosis.

We used a logistic regression model to assess risk factors associated with longer episodes of depression, anxiety, or both ( $\geq 90$ days) occurring at any time during the study.

For patients who had a recurrence, we used the log rank test to look at predictors of depression, anxiety, or both after recurrence. The variables we considered were psychological history, presence of an intimate confiding relationship, an episode of depression, anxiety, or both around diagnosis or any time up to recurrence, and age and time to recurrence. Owing to insufficient numbers, we considered these factors in univariate analysis only.

We tested inter-rater reliability on a random sample of 10 interviews. Agreement was $100 \%$ on presence of an episode of depression, anxiety, or both; $92 \%$ on severity ratings given to life events (weighted $\kappa=0.75$ ), and $91 \%$ on severity ratings given to difficulties (weighted $\kappa=0.63$ ).

\section{Results}

Overall, 91\% (202 of 222) of eligible women completed the first interview; 15 declined to participate, two moved away from the area after their primary diagnosis, and three were not contactable. Of the 202 women initially interviewed, nine declined the
Table 1 Baseline personal and clinical characteristics of women with first diagnosis of breast cancer. Values are numbers (percentages) unless stated otherwise

\begin{tabular}{|c|c|}
\hline Variable & Women with breast cancer ( $\mathrm{n}=202)$ \\
\hline Mean (SD) age & $48.4(7.8)$ \\
\hline \multicolumn{2}{|l|}{ Marital status: } \\
\hline Married or cohabiting & $151(75)$ \\
\hline Divorced or separated & $25(12)$ \\
\hline Widowed & $8(4)$ \\
\hline Single & $18(9)$ \\
\hline \multicolumn{2}{|c|}{ Intimate confiding relationship: } \\
\hline Present & $134(66)$ \\
\hline Absent & $68(34)$ \\
\hline \multicolumn{2}{|c|}{ Previous psychological treatment: } \\
\hline None & $148(73)$ \\
\hline Some & $54(27)$ \\
\hline \multicolumn{2}{|c|}{ No of axillary lymph nodes affected: } \\
\hline None & $81(40)$ \\
\hline $1-3$ & $82(41)$ \\
\hline $4-9$ & $29(14)$ \\
\hline$\geq 10$ & $10(5)$ \\
\hline \multicolumn{2}{|l|}{ Tumour histology: } \\
\hline Ductal grade III & $64(32)$ \\
\hline Other & $138(68)$ \\
\hline \multicolumn{2}{|l|}{ Tumour size $(\mathrm{cm})$ : } \\
\hline$\leq 2$ & $94(47)$ \\
\hline $2-5$ & $81(40)$ \\
\hline$\geq 5$ & $21(10)$ \\
\hline Not known & $6(3)$ \\
\hline \multicolumn{2}{|l|}{ Adjuvant treatment: } \\
\hline None & $12(6)$ \\
\hline Endocrine therapy & $96(48)$ \\
\hline Chemotherapy & $89(44)$ \\
\hline Both & $4(2)$ \\
\hline Not known & $1(0.5)$ \\
\hline
\end{tabular}

second interview, seven the third interview, and one the fourth interview. A further seven women moved away from the area before the second interview, three before the third interview, and two before the fourth interview. Three women died of causes not directly related to breast cancer. In total, $77 \%$ (170 of the original 222) of the women provided complete interview data either up to five years after diagnosis or to recurrence. Recurrence was confirmed in 39 women. Table 1 lists the personal and clinical characteristics of the women.

\section{Prevalence of and risk factors for depression and anxiety}

Point prevalence of depression, anxiety, or both (including borderline cases) was 33\% at diagnosis and $24 \%$ at three months after diagnosis, dropping to $15 \%$ at one year (figure). The annual prevalences for the first to fifth years after diagnosis were $48 \%$, $25 \%, 23 \%, 22 \%$, and $15 \%$, respectively.

Previous psychological treatment was associated with depression, anxiety, or both around diagnosis (table 2). For the medium term, risk factors were previous psychological treatment, lack of an intimate confiding relationship, and experience of severe non-cancer difficulties. In the longer term, depression and anxiety were associated with the lack of an intimate confiding relationship, younger age, severe non-cancer difficulties, and an earlier episode of depression, anxiety, or both after diagnosis. Treatment and prognostic factors, comprising number of axilliary lymph nodes involved, tumour size and histology, and type of adjuvant treatment, were not associated with depression and anxiety at any time. These were excluded from the multivariate model. 


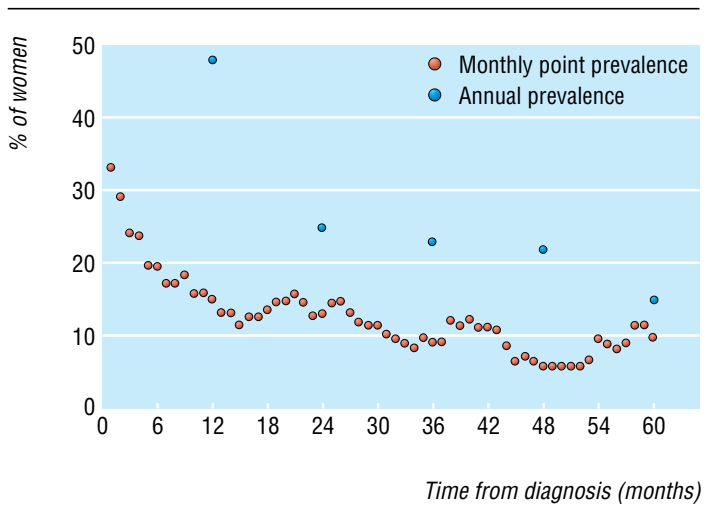

Prevalence of episodes of depression, anxiety, or both in women with diagnosis of early breast cancer

\section{Duration of episodes of depression and anxiety}

Overall, $81(40 \%)$ women reported an episode of depression, anxiety, or both lasting at least 90 days, $41(20 \%)$ reported an episode lasting fewer than 90 days, and $80(40 \%)$ reported no episodes (131 of these 202 women were followed up for five years). Previous psychological treatment (odds ratio 2.90, 95\% confidence interval 1.25 to 6.75 ) and lack of an intimate confiding relationship (2.34, 1.14 to 4.82$)$ were significantly associated with longer episodes ( $>90$ days) of depression, anxiety, or both rather than shorter $(<90$ days $)$ ones.

\section{Prevalence of, and risk factors for, depression and anxiety at recurrence}

Of the 39 patients who had a recurrence within five years of diagnosis, we had data for 33 . Depression and anxiety were more prevalent after recurrence than after the initial diagnosis. Overall, $45 \%$ (95\% confidence interval $28 \%$ to $64 \%$ ) of women had an episode of depression, anxiety, or both in the three months after diagnosis of recurrence compared with 36\% (30\% to $43 \%$ ) in the three months after initial diagnosis. We found no significant association between depression and anxiety at recurrence and any of the risk factors included in the study, including an episode of depression, anxiety, or both around diagnosis. The duration between initial diagnosis and recurrence was not a risk factor.

\section{Discussion}

In women with early breast cancer, the prevalence of depression, anxiety, or both in the year after diagnosis is around twice that of the general female population. Thereafter, women in remission show similar levels of depression and anxiety to the general female population, but those with recurrence of disease experience a sharp increase in levels. The risk factors for depression and anxiety are related to the patient rather than to disease or treatment.

Our large cohort study is unique in that we used structured interviews and standardised diagnostic criteria to assess the point prevalence and period prevalence of clinically important depression and anxiety in women with early breast cancer in the five years after diagnosis. We also used a standardised interview method to examine the broader social context in which cancer occurred.

The point prevalence of depression, anxiety, or both in the year after diagnosis was lower than reported in previous studies, ${ }^{78}$ including one study carried out in the same breast unit as our study. The effect of diagnosis and treatment may have been reduced because of improvements in survival rates from breast cancer and better supportive care, including management of the side effects of anticancer treatments. The stigma associated with breast cancer may have decreased over time and a diagnosis may be less likely to provoke clinically important distress. Alternatively, the use of different diagnostic criteria for depression and anxiety may have contributed to the different levels recorded in our study. ${ }^{10}$

Our findings are in line with other studies, which show that factors related to the patient rather than to the disease or treatment increase the risk of clinically important depression and anxiety in women who remain free of breast cancer in the year after the disease is diagnosed. ${ }^{11-13}$ These risk factors include the main ones for depression and anxiety in the general female population $^{5}$-namely, younger age, previous psychological problems, non-cancer related difficulties, and lack of social support. The quality of social support predicts better health related quality of life generally in women who are in remission from breast cancer. ${ }^{2}$ Effective dissemination of information and the communication style of the surgeon seem to protect women against depression, anxiety, or both, ${ }^{11} 13$ whereas offering women choice of surgical treatment does not. ${ }^{814}$ The risk is not affected by clinical factors such as prognosis, type of surgery, or adjuvant radiotherapy. ${ }^{15-18}$ Adjuvant chemotherapy may increase the risk of depression, anxiety, or both during but not after treatment. ${ }^{7} 18$ Detecting breast cancer through screening rather than through self discovery does not seem to increase the risk of depression and anxiety. ${ }^{15} 19$

The increased levels of depression and anxiety around recurrence of breast cancer highlight the adverse effect of this event on women's mental health, which signals incurability and possi-

Table 2 Risk factors for depression, anxiety, or both in phase around diagnosis and medium and longer term

\begin{tabular}{|c|c|c|c|c|c|c|}
\hline \multirow[b]{2}{*}{ Risk factors } & \multicolumn{2}{|c|}{$\begin{array}{c}\text { Phase around diagnosis (-1 month to } 4 \\
\text { months) }\end{array}$} & \multicolumn{2}{|c|}{ Medium term (4 months to 2 years) } & \multicolumn{2}{|c|}{ Longer term (2 years to 5 years) } \\
\hline & Odds ratio* $(95 \% \mathrm{Cl})$ & $P$ value & Hazard ratio† $(95 \% \mathrm{Cl})$ & $P$ value & Hazard ratio† $(95 \% \mathrm{CI})$ & $P$ value \\
\hline Past psychological treatment & $1.90 \ddagger(0.99$ to 3.66$)$ & 0.05 & $1.38 \neq(1.10$ to 1.74$)$ & $<0.01$ & - & - \\
\hline $\begin{array}{l}\text { Lack of intimate confiding } \\
\text { relationship }\end{array}$ & 1.67 (0.91 to 3.01$)$ & 0.10 & $1.38 \ddagger$ (1.11 to 1.72$)$ & $<0.01$ & $1.43 \ddagger$ (1.11 to 1.86$)$ & $<0.01$ \\
\hline Younger age§ & 0.98 (0.94 to 1.01$)$ & 0.18 & 0.98 (0.96 to 1.00$)$ & 0.10 & $0.96 \ddagger(0.93$ to 0.99$)$ & $<0.01$ \\
\hline Severe life events & 1.02 (0.51 to 2.04$)$ & 0.95 & 0.99 (0.73 to 1.35$)$ & 0.76 & 1.54 (0.81 to1.47) & 0.55 \\
\hline Severe difficulties & 0.95 (0.49 to 1.83$)$ & 0.95 & $1.36 \mp(1.05$ to 1.76$)$ & 0.02 & $1.54 \ddagger(1.14$ to 2.09$)$ & $<0.01$ \\
\hline $\begin{array}{l}\text { Previous episode of depression } \\
\text { or anxiety in study period }\end{array}$ & NA & NA & - & - & $1.55 \ddagger$ (1.17 to 2.06$)$ & $<0.01$ \\
\hline
\end{tabular}

$\mathrm{NA}=$ not available. Only variables indicating significance at univariate level $(P \leq 0.1)$ were included in multivariate model.

*Logistic regression.

tCox's proportional hazards model

‡Significant at $5 \%$ level.

$\S 0$ dds ratio and hazards ratio for one year change in age. 


\section{What is already known on this topic}

More women are surviving breast cancer because of early detection and improved treatment

Around twice as many of these women than the general female population have clinically important depression, anxiety, or both in the year after diagnosis

Less is known about the prevalence of, and risk factors for, clinically important depression and anxiety beyond the year after diagnosis

\section{What this study adds}

After the first year following a diagnosis of breast cancer, women in remission have levels of depression and anxiety that are comparable with those of the general female population

The risk factors for depression and anxiety in the five years after diagnosis are related to the patient rather than to the disease or its treatment

Psychological interventions for women with breast cancer should take account of the broader social context in which the cancer occurs, focusing on improved social support

ble physical burden. Much less is known about the psychological problems experienced by women with advanced breast cancer. The improved survival of women with advanced disease from new treatments increases the need to understand better these issues. ${ }^{20}{ }^{21}$ The lack of risk factors for depression and anxiety at recurrence suggest that the adverse psychological consequences are relatively independent of an individual's own vulnerability factors. ${ }^{22}{ }^{23}$ These findings should be interpreted with caution, however, because of the small sample sizes.

We examined clinically important anxiety and depression, but this is only one dimension of the psychological consequence of survival after breast cancer is diagnosed. The absence of problems from anxiety and depression after the first year of diagnosis does not imply the absence of other psychological difficulties for women in remission from breast cancer. These include more subtle and existential concerns about loss of hope and certainty about the future.

Our findings are relevant to the implementation of the guidance for improving supportive and palliative care services for adults with cancer commissioned by the National Institute for Clinical Excellence. ${ }^{24}$ Effective psychological services are needed for women with breast cancer, especially in the year after diagnosis and around recurrence. We also found that women who are free of the disease but who are at risk of developing depression and anxiety are likely to benefit from psychological interventions that take account of the broader social context in which cancer occurs, including interventions to improve social support. Improving social support may also limit chronic depression, anxiety, or both in those women who lack an intimate confiding relationship.

We thank Tirril Harris for advice on the life events and difficulties schedule, Karen Pinder for undertaking some of the research interviews, the women who participated in the study, and Ian Fentiman who had clinical responsibility for the women.

Contributors: $\mathrm{CB}$ coordinated the study, acquired and interpreted the data, and drafted the paper. AR and MR conceived and designed the study; AR will act as guarantor. VC and SL conducted the statistical analysis. JG acquired the data. All authors critically revised the paper.

Funding: Cancer Research UK.
Competing interests: None declared.

Ethical approval: Guy's Hospital research ethics committee.

1 Peto R, Boreham J, Clarke M, Davies C, Beral V. UK and USA breast cancer deaths down 25\% in year 2000 at ages 20-69 years. Lancet 2000;355:1822.

2 Ganz P, Desmond K, Leedham B, Rowland J, Meyerowitz B, Belin T. Quality of life in long-term, disease-free survivors of breast cancer: a follow-up. J Nat Cancer Inst 2002;94:39-49.

3 Spitzer R, Williams J, Gibbon M, First M. Structured clinical interview for DSM III-R (SCID-P,version 1.0). Washington DC: American Psychiatric Press, 1990.

4 American Psychiatric Association, Committee on Nomenclature and Statistics. Diagnostic and statistical manual of mental disorders, revised 3rd ed. Washington, DC: APA, 1987.

5 Brown G, Harris T. Social origins of depression: a study of psychiatric disorder in women. London: Tavistock, 1978.

6 O'Connor P, Brown G. Supportive relationships: fact or fancy? J Soc Pers Rels 1984;1:159-75.

7 Dean C. Psychiatric morbidity following mastectomy: preoperative predictors and types of illness. J Psychosom Res 1987:31:3, 385-92

8 Fallowfield L, Hall A, Maguire G, Baum M. Psychological outcomes of different treatment policies in women with early breast cancer outside a clinical trial. BMJ 1990;301:575-80.

9 Ramirez A, Richards M, Jarrett S, Fentiman I. Can mood disorder in women with breast cancer be identified preoperatively? Br J Cancer 1995;72:1509-12.

10 Chochinov HM, Wilson KG, Enns M, Lander S. Prevalence of depression in the terminally ill: effects of diagnostic criteria and symptom threshold judgments. Am J Psychiatry 1994;151:537-40.

11 Harrison J, Maguire P. Predictors of psychiatric morbidity in cancer patients. $\mathrm{Br} J$ Psychiatry 1994;165:593-8.

12 Pinder KL, Ramirez AJ, Richards MA, Gregory WM. Cognitive responses and psychiatric disorder in women with operable breast cancer Psycho-Oncology 1994;3:129-37.

13 Fallowfield L. Effects of breast conservation on psychological morbidity associated with diagnosis and treatment of early breast cancer. BMJ 1986;293:1331-4.

14 fiagnosis and treatment of early breast cancer. BMJ 1986;293:1331-4. Fallowfield LJ, Hall A, Maguire P, Baum M, A'Hern RP. Psychol
offered choice of surgery for breast cancer. BMJ 1994;309:448.

15 Burgess C, Ramirez A, Richards M, Potts HWW. Does the method of detection of breast cancer affect subsequent psychiatric morbidity? Eur J Cancer 2002;38:1622-5.

16 Kiebert G, de Haes J, Van de Velde C. The impact of breast conserving treatment and mastectomy on the quality of life of early stage breast cancer patients: a review. J Clin Oncol 1991;9:1059-70.

17 Lee M, Love S, Mitchell J, Parker E, Rubens R, Watson J, et al. Mastectomy or conservation for early breast cancer: psychological morbidity. Eur J Cancer 1992;28:1340-4.

18 Hughson A, Cooper A, McArdle C, Smith D. Psychological impact of adjuvant chemotherapy in the first two years after mastectomy. BMJ 1986;293:1268-72.

19 Haddad P, Maguire P, Jones B. Effect of mode of breast cancer diagnosis on subsequent affective disorder. Breast 1994:3:218-21.

20 O'Shaughnessy J, Miles D, Vukelja S, Moiseyenko V, Ayoub JP, Cervantes G, et al. Superior survival with Capecitabine plus Docetaxel combination therapy in Anthracyclinepretreated patients with advanced breast cancer: phase III trial results. J Clin Oncol 2002;20:2812-23.

21 Chia SKL, Speers C, Kang A, D'Yachkova Y, Malfair Taylor S, Barnett J, et al. The impact of new chemotherapeutic and hormonal agents on the survival of women with metastatic breast cancer (MBC) in a population based cohort. Proc Am Soc Clin Oncol 2003;22:6.

22 Hall A, Fallowfield L, A'Herne R When breast cancer recurs: a three-year prospective study of psychiatric morbidity. Breast 1996;2:197-201.

23 Okamura H, Watanabe T, Narabayashi M, Katsumata N, Ando M, Adachi I, et al. Psychological distress following first recurrence of disease in patients with breast canPsychological distress following first recurrence of disease in patient
cer: prevalence and risk factors. Breast Canc Res Treat 2000;61:131-7.

24 National Institute for Clinical Excellence. Supportive and palliative care for people with National Institute for Clinical
cancer. London: NICE, 2004.

Accepted 20 December 2004

doi 10.1136/bmj.38343.670868.D3

Cancer Research UK London Psychosocial Group, Institute of Psychiatry, King's College London, St Thomas's Hospital, London SE1 7EH

College London, St Thomas's Hospist
Caroline Burgess health psychologist

Amanda Ramirez professor of liaison psychiatry

Jill Graham health psychologist

Michael Richards national cancer director

Cancer Research UK Medical Statistics Group, Centre for Statistics in Medicine, Institute of Health Sciences, Headington, Oxford OX3 7LF

Victoria Cornelius medical statistician

Sharon Love medical statistician

Correspondence to: C Burgess caroline.burgess@kcl.ac.uk

\section{Amendment}

This is version 2 of the paper. In this version the title has been corrected. It now reads as Anxiety and depression [in the previous version it was Anxiety, depression, or both] in women with early breast cancer: five year observational cohort study. 